\title{
Safety and efficacy of ultrasound guided corticosteroid injections into temporomandibular joints in children with active juvenile idiopathic arthritis
}

\author{
S Habibi, J Ellis, H Strike, AV RamananPediatric Rheumatology \\ From 18th Pediatric Rheumatology European Society (PReS) Congress \\ Bruges, Belgium. 14-18 September 2011
}

\section{Background}

Prevalence of temporomandibular arthritis in JIA varies widely, reported rates ranging from $17-87 \%$. Untreated inflammation with joint destruction can lead to asymmetrical mandibular growth with jaw deviation, dental malocclusion and micrognathia. Intra-articular steroid injection for TMJ arthritis has been found to be effective. There are limited reports on the efficacy of ultrasound guided steroid injections of the TMJ's in JIA.

\section{Aim}

To assess the safety and efficacy of ultrasound guided corticosteroid injection, done by a paediatric rheumatologist, into the temporomandibular joints in children with JIA.

\section{Methods}

Children with JIA presenting to rheumatology clinic assessed for TMJ arthritis. Triamcinolone hexacetonide injected in those with active arthritis assessed by MRI, using ultrasound guidance under general anaesthesia by a single paediatric rheumatologist trained in procedure. Efficacy and safety was assessed post-injection by patient guided symptoms and physical examination.

Table 1 Improvement in symptoms

\begin{tabular}{ll}
\hline Symptom & Improvement [n (\%)] \\
\hline Pain (17) & $17(100 \%)$ \\
\hline Jaw deviation (14) & $13(92.8 \%)$ \\
\hline Chewing dysfunction(7) & $5(71.4 \%)$ \\
\hline
\end{tabular}

\section{Results}

38 children (34 girls) with TMJ injection between Jan 2009-Jan 2011 studied. Mean age: 12.25 \pm 3.55 years (range $=5-18$ years). Mean disease duration: $4.54 \pm 2.73$ years (1.5-11.1years). Symptoms pre-injection: pain:17/ 38(44.7\%), jaw deviation:14/38(36.8\%), restricted jaw movement:13/38(34.2\%), chewing dysfunction:7/38 (18.4\%), micrognathia:5(12.5\%). Total 63 joints injected. Injection efficacious:58/63(92.06\%) joints(Table 1). Stiffness was persistent in 2 children with both TMJ's injected \& 1 had persistent jaw deviation. Injection site scar in 1 child.

\section{Conclusions}

Ultrasound guided corticosteroid injection into the temporomandibular joint done by a paediatric rheumatologist trained in the procedure safe with a high rate of success.

Published: 14 September 2011

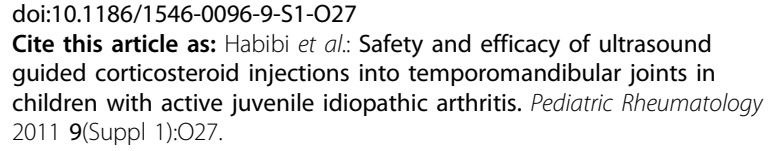

